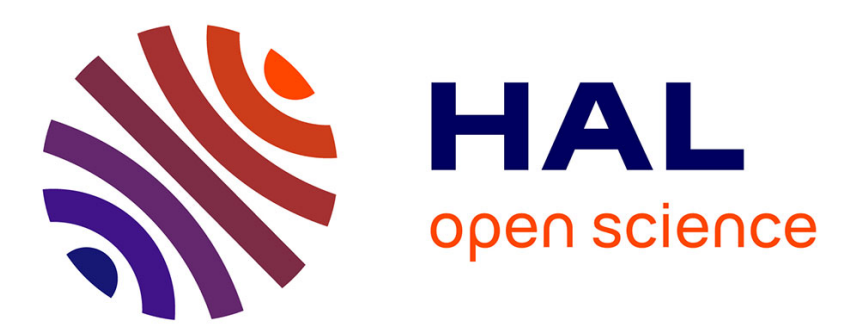

\title{
RMT for whitening space correlation and applications to radar detection
}

\author{
Romain Couillet, Maria S Greco, Jean-Philippe Ovarlez, Frédéric Pascal
}

\section{To cite this version:}

Romain Couillet, Maria S Greco, Jean-Philippe Ovarlez, Frédéric Pascal. RMT for whitening space correlation and applications to radar detection. IEEE 6th International Workshop on Computational Advances in Multi-Sensor Adaptive Processing (CAMSAP 2015), Dec 2015, Cancun, Mexico. pp.149 - 152, 10.1109/CAMSAP.2015.7383758 . hal-01262631

\section{HAL Id: hal-01262631 \\ https://hal.science/hal-01262631}

Submitted on 26 Jan 2016

HAL is a multi-disciplinary open access archive for the deposit and dissemination of scientific research documents, whether they are published or not. The documents may come from teaching and research institutions in France or abroad, or from public or private research centers.
L'archive ouverte pluridisciplinaire HAL, est destinée au dépôt et à la diffusion de documents scientifiques de niveau recherche, publiés ou non, émanant des établissements d'enseignement et de recherche français ou étrangers, des laboratoires publics ou privés. 


\title{
RMT for Whitening Space Correlation and Applications to Radar Detection
}

\author{
Romain Couillet*, Maria S. Greco ${ }^{\dagger}$, Jean-Philippe Ovarlez ${ }^{\ddagger}$ and Frédéric Pascal* \\ ${ }^{*}$ L2S/CentraleSupélec-CNRS-Université Paris-Sud, 3 rue Joliot-Curie, 91190 Gif-sur-Yvette, France \\ (e-mail: romain.couillet, frederic.pascal @ centralesupelec.fr) \\ $\dagger$ Dipartimento di Ingegneria dell'Informazione, University of Pisa, Pisa 56122, Italy \\ (e-mail: m.greco@iet.unipi.it) \\ $\ddagger$ ONERA, DEMR/TSI, Chemin de la Hunière, 91120 Palaiseau, France and SONDRA/CentraleSupélec \\ (e-mail: jean-philippe.ovarlez@onera.fr)
}

\begin{abstract}
Adaptive radar detection and estimation schemes are often based on the independence of the secondary data used for building estimators and detectors. This paper relaxes this constraint and deals with the non-trivial problem of deriving detection and estimation schemes for joint spatial and temporal correlated radar measurements. Latest results from Random Matrix theory, used for large dimensional regime, allows to build a Toeplitz estimate of the spatial covariance matrix while the temporal covariance matrix is then estimated in a conventional way (Sample Covariance Matrix, M-estimates). These two joint estimates of the spatial and temporal covariance matrices leads to build Adaptive Radar Detectors, like Adaptive Normalized Matched Filter (ANMF). We show that taking care of the spatial covariance matrix may lead to significant performance improvements compared to classical procedures.
\end{abstract}

\section{INTRODUCTION}

In many applications, data can be viewed as a joint spatial and temporal process. In radar and imagery applications, taking into account this constraint can be of high interest. For high resolution radar, the sea clutter is clearly jointly spatially and temporally correlated. In mutichannel (polarimetric, interferometric or multi-temporal) SAR imaging, the multivariate vector characterizing each spatial pixel of the image is correlated over the channels but can also be strongly correlated with those of neighborhood pixels. In the radar community, one generally supposes that the vectors of information collected over a spatial support are identically and independently distributed. This strong assumption allows to build easily Maximum Likelihood Estimators of parameters like for example, covariance matrix required for adaptive detection leading to overestimated performance of such detectors. The aim of this proposal is to relax this hypothesis through the use of recent Random Matrix Theory results. This could help in many radar applications.

Covariance estimation is a fundamental problem in multivariate statistics. Many techniques for hypothesis testing, inference, denoising and prediction in speech, radar, wireless communication and finance applications (just to quote a few) rely on accurate estimation of the true covariance matrix or the scatter matrix.
To estimate the scatter matrix (or a sub-set of its elements) of any observed vector under test $\mathbf{y}$ (primary vector on dimension $N$ ), generally it is supposed to dispose of $K>N$ secondary vectors, independent and identically distributed (IID), that share with the primary vector the same statistical characteristics [1], [2]. In some applications, as in high spatial resolution radars, the hypothesis of independence (or even of uncorrelation) of the secondary vectors is seldom satisfied due to the nature of the phenomenon at hand. Particularly, for target detection purposes, due to the lack of knowledge of the spectral characteristic of the clutter and the variability of it on long period of time and large surfaces, the covariance matrix of the clutter must be estimated and plugged into adaptive detectors in both cases of Gaussian and non-Gaussian distributed disturbance.

In this case the secondary vectors used for matrix estimation contain the samples of the echoes backscattered by the range cells surrounding the cell under test (CUT). If the dimension of the range cells is of the order of meters or even less, particularly in the case of sea clutter, some correlation among echoes coming from adjacent range cells has been observed, as shown in figure 2 [3] where the estimated space correlation coefficient of sea clutter data, recorded by IPIX radar [4], is illustrated for different range resolutions. The periodicity, particularly evident for a resolution of 3 meters is due to the periodicity of the sea surface and of the sea wave behavior. Higher the radar range resolution, shorter the waves that can be resolved by the radar itself (in the figure each dot corresponds to a different range cell). The correlation does not last for kilometers but it is enough to affect the covariance matrix estimation and the performance of the adaptive detectors.

To fill this gap, this paper presents an efficient way of estimating first the correlation matrix from the samples in order to whiten this space dependency. More precisely, based on recent results from Random Matrix Theory (RMT), a consistent estimate of the correlation matrix is introduced, which allows to remove this correlation. Then, a classical detection test, namely the Adaptive Normalized Matched Filter 


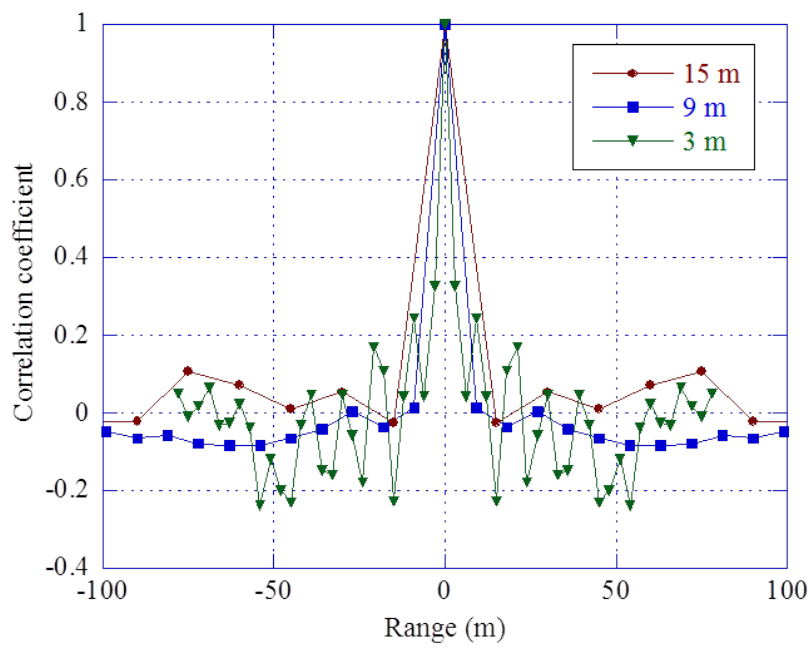

Fig. 1. Sea clutter spatial correlation, IPIX radar [3]

(ANMF), is analyzed in order to underline th eimprovement brought by the proposed approach.

The paper is organized as follows: Section III introduces the problem formulation while the main contribution is contained in Section IV. Then, Section V presents detection performance obtained Monte-Carlo simulations, that enlighten the interest of the proposed approach. Finally, Section ?? draws some conclusions and perspectives.

Notations : vectors and matrix are in boldface, matrix in capitals and vectors in small letters, ${ }^{H}$ the Hermitian operator and ${ }^{T}$ the transpose.

\section{PROBLEM FORMULATION}

Detecting a complex signal corrupted by an additive Gaussian noise $\mathbf{c} \sim \mathcal{C} \mathcal{N}(\mathbf{0}, \mathbf{M})$ in a $N$-dimensional complex observation vector $\mathbf{y}$ can be stated as the following binary hypothesis test:

$$
\left\{\begin{array}{lll}
H_{0}: \mathbf{y}=\mathbf{c} & \mathbf{y}_{i}=\mathbf{c}_{i} \quad i=1, \ldots, K \\
H_{1}: \mathbf{y}=\alpha \mathbf{p}+\mathbf{c} & \mathbf{y}_{i}=\mathbf{c}_{i} \quad i=1, \ldots, K
\end{array},\right.
$$

where $\mathbf{p}$ is a perfectly known complex steering vector, $\alpha$ is the unknown signal amplitude and where the $\mathbf{c}_{i} \sim \mathcal{C N}(\mathbf{0}, \mathbf{M})$ are $K$ signal-free non independent measurements, traditionally called the secondary data, used to estimate the background covariance matrix $\mathbf{M}$. To model the spatial dependency between the secondary data, from the Gaussian assumption on $\mathbf{c}_{i}$, we may write $\mathbf{C}=\left[\mathbf{c}_{1}, \ldots, \mathbf{c}_{K}\right]$ under the following form.

Assumption 1 (Time dependence) For $\mathbf{C}=\left[\mathbf{c}_{1}, \ldots, \mathbf{c}_{K}\right]$,

$$
\mathbf{C}=\mathbf{M}^{1 / 2} \mathbf{X} \mathbf{T}^{1 / 2},
$$

where $\mathbf{M} \in \mathbb{C}^{N \times N}$ and $\mathbf{T} \in \mathbb{C}^{K \times K}$ are both nonnegative definite, $\mathbf{X}$ is standard Gaussian, and $\mathbf{T}$ satisfies the normalization $\frac{1}{K} \operatorname{tr} T=1$.
We shall need the following technical assumptions.

Assumption 2 (Norm boundedness of $\mathbf{M}$ ) We have

$$
\limsup _{N \rightarrow \infty}\|\mathbf{M}\|<\infty
$$

where the assumed norm is the spectral norm.

Assumption 3 (Toeplitz Structure for $\mathbf{T}$ ) The matrix $\mathbf{T}$ is Toeplitz, i.e., for all $i, j, \mathbf{T}_{i, j}=t_{|i-j|}$ for $t_{0}=1$ and $t_{k} \in \mathbb{C}$, and positive definite. Besides, $\sum_{k=0}^{\infty}\left|t_{k}\right|<\infty$.

Upon Assumption 3, it can be shown that $\lim \sup _{K \rightarrow \infty}\|\mathbf{T}\|<\infty$.

\section{CONTRIBUTIONS}

The following technical results unfold directly from a careful check of the results of [5].

Proposition 1 (Consistent Estimation for T) As $N, K \rightarrow$ $\infty$ such that $N / K \rightarrow c \in(0, \infty)$, and for every $\beta<1$,

$$
N^{\beta}\left\|\mathcal{T}\left[\frac{1}{N} \mathbf{C}^{H} \mathbf{C}\right]-\left(\frac{1}{N} \operatorname{tr} \mathbf{M}\right) \mathbf{T}\right\| \rightarrow 0
$$

almost surely, where the norm is the matrix operator norm and $\mathcal{T}[\cdot]$ is the Toeplitzification operator defined by $(\mathcal{T}[\mathbf{X}])_{i j}=$ $\frac{1}{K} \sum_{k=1}^{K} \mathbf{X}_{k, k+|i-j|}$.

Proof: It suffices to update [5, Lemmas 3-6] by adding the contribution of the matrix $\mathbf{M}$. Notably, the upper bound $\limsup _{K \rightarrow \infty}\|\mathbf{M}\|$ on the norm of $\mathbf{M}$ will introduce a supplementary term, which shall translate in [5, Theorem 2] into multiplying the denominator of the exponential term by $\lim \sup _{K \rightarrow \infty}\|\mathbf{M}\|$.

Thus, despite the joint growth rate $N, K \rightarrow \infty$ which is usually detrimental to consistently estimating $\mathbf{T}$ in nonparametric settings (as proved by random matrix theory), Proposition 1 ensures that the Toeplitz structure of $\mathbf{T}$ is strong enough constraint to recover consistency. The matrix $\mathbf{M}$ here appears not to alter the result, mostly because $\mathbf{X}$, being white Gaussian, is left-unitarily invariant.

Denote now $\hat{\mathbf{M}}$ the following time-whitened sample covariance matrix of $c_{1}, \ldots, c_{N}$ :

$$
\hat{\mathbf{M}} \triangleq \frac{1}{K} \mathbf{C}\left(\mathcal{T}\left[\frac{1}{N} \mathbf{C}^{H} \mathbf{C}\right]\right)^{-1} \mathbf{C}^{H}
$$

For technical reasons, we shall demand the following additional assumption.

\section{Assumption 4 (Boundedness of $\mathbf{T}$ away from zero) For} $\lambda_{1}(\mathbf{T}) \leq \ldots \leq \lambda_{K}(\mathbf{T})$

$$
\liminf _{K} \lambda_{1}(\mathbf{T})>0 .
$$

Then, as a corollary of Proposition 1, we find that, as $N, K \rightarrow \infty$ with $N / K \rightarrow c \in(0, \infty)$,

$$
N^{\beta}\left\|\hat{\mathbf{M}}-\frac{\check{\mathbf{M}}}{\frac{1}{N} \operatorname{tr} \mathbf{M}}\right\| \rightarrow 0
$$


almost surely for each $\beta<1$, where $\check{\mathbf{M}} \triangleq \frac{1}{N} \mathbf{M}^{\frac{1}{2}} \mathbf{X} \mathbf{X}^{H} \mathbf{M}^{\frac{1}{2}}$. We thus asymptotically recover a normalized version of the time-uncorrelated sample covariance matrix. Since any $\beta<1$ is allowed, this implies that most functionals of $\hat{\mathbf{M}}$ having fluctuations at a rate lower than $N^{-\beta}, \beta<1$, have the same behavior as the traditional time-uncorrelated (inaccessible) sample covariance estimator $\frac{1}{N} \mathbf{M}^{\frac{1}{2}} \mathbf{X} \mathbf{X}^{H} \mathbf{M}^{\frac{1}{2}}$, up to a constant.

As an immediate application of the above, define the adaptive normalized matched filter (ANMF) [6] detector $T_{N} \underset{H_{1}}{\stackrel{H_{0}}{\lessgtr}} \gamma$ to be based on the following statistics:

$$
T_{N} \triangleq \frac{\left|y^{H} \hat{\mathbf{M}}^{-1} p\right|}{\sqrt{y^{H} \hat{\mathbf{M}}^{-1} y} \sqrt{p^{H} \hat{\mathbf{M}}^{-1} p}} .
$$

Since $T_{N}$ is clearly invariant by scalar multiplications of $\hat{\mathbf{M}}$, exploiting standard results from random matrix theory, it then comes that, as $N, K \rightarrow \infty$ with $N / K \rightarrow c \in(0,1)$,

$$
N^{\beta}\left(T_{N}-\check{T}_{N}\right) \rightarrow 0
$$

almost surely, where $\check{T}_{N}$ is the (inaccessible) test

$$
\check{T}_{N} \triangleq \frac{\left|y^{H} \check{\mathbf{M}}^{-1} p\right|}{\sqrt{y^{H} \check{\mathbf{M}}^{-1} y} \sqrt{p^{H} \check{\mathbf{M}}^{-1} p}} .
$$

In particular, for $\Gamma \in \mathbb{R}$, it is known from [7], that, as $K, N$ grow large, the ANMF provides non trivial results when setting thresholds $\gamma=\frac{\Gamma}{\sqrt{N}}$, i.e., the false alarm rate

$$
\mathrm{P}\left(T_{N}>\frac{\Gamma}{\sqrt{N}}\right)
$$

converges, under the $H_{0}$ hypothesis for $y$, towards a limit in $(0, \infty)$. Similarly, assuming $\alpha=\frac{A}{\sqrt{N}}$ for finite $A$ in (??), the probability of correct detection leads to non trivial results when $\gamma=\frac{\Gamma}{\sqrt{N}}$. From our previous reasoning, and the effective speed of $N^{\beta}, \beta<1$, of our estimator, it then comes the following result.

Corollary 1 (ANMF Performance) Both under $H_{0}$ and $H_{1}$, as $N, K \rightarrow \infty$ with $N / K \rightarrow c \in(0,1)$,

$$
\mathrm{P}\left(T_{N}>\frac{\Gamma}{\sqrt{N}}\right)-\mathrm{P}\left(\check{T}_{N}>\frac{\Gamma}{\sqrt{N}}\right) \rightarrow 0 .
$$

Proof: This follows from the fact that $\sqrt{N}\left(T_{N}-\check{T}_{N}\right) \rightarrow 0$ almost surely.

Thus, we have shown that the proposed ANMF detector $T_{N}$ is consistent with the ideal, but unobservable, timeuncorrelated detector $\check{T}_{N}$.

\section{NUMERICAL RESULTS}

This section is devoted to the Monte-Carlo simulations in order to compare the proposed approach, i.e. a space correlation whitening, with the classical approach. For that purpose, we consider the model given by equation (2). The settings are the following: the covariance matrix $\mathbf{M}$ is a full Toeplitz matrix given by its elements $M_{i j}=\rho_{\mathrm{M}}^{|i-j|}$ for $i, j=1, \ldots, N$ and the (space) correlation matrix $\mathbf{T}$ is also a Toeplitz matrix with 3 non-null elements (5 nonnull diagonals), i.e. $T_{i j}=\rho_{\mathbf{T}}^{|i-j|}$ for $i, j=1, \ldots, K$. The parameters are given by $N=10, K=20, \rho_{\mathbf{M}}=0.5$ and $\rho_{\mathbf{T}}=0.9$, so that the covariance matrix is the identity matrix. Then, we have plotted the Probability of False Alarm (PFA) versus the detection threshold and also the detection performance, namely the Probability of Detection (PD) versus the Signal-to-Noise Ratio (SNR).

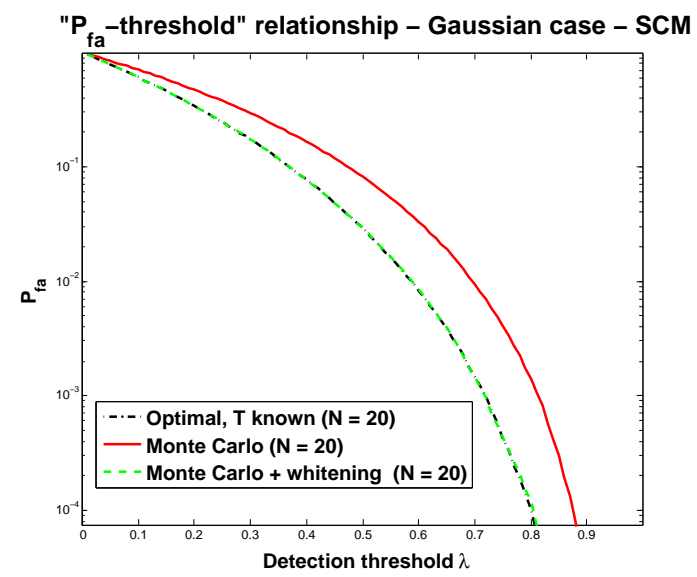

(a) SCM

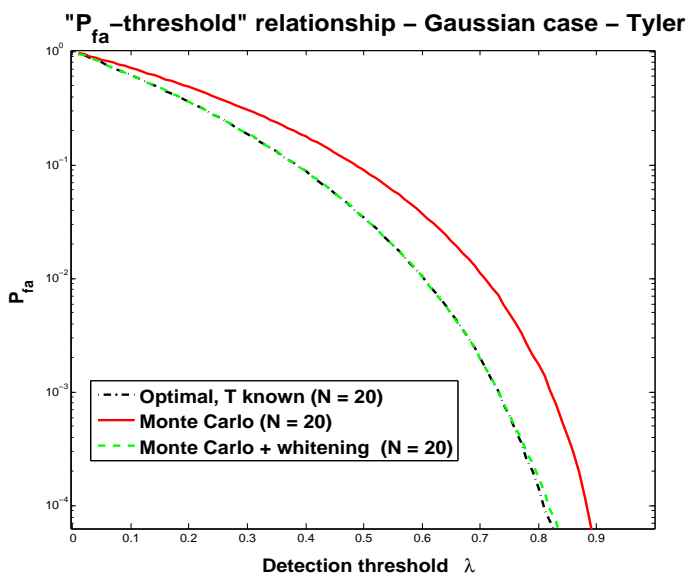

(b) Tyler

Fig. 2. PFA-Threshold relationship under Gaussian noise, $N=10, K=20$.

To evaluate the gain of the method, we have compared three scenarios:

- the case where $\mathbf{T}$ is assumed to be known and can be removed. Thus, the covariance estimate is given by $\check{\mathbf{M}}$, and the corresponding test statistic is $\check{T}_{N}$ (eq. (5)),

- the proposed estimate, given by equation (3), and the corresponding test statistic $T_{N}$ (eq. (4))

- the classical approach that does not take into account the space correlation, that is the covariance estimator is given by

$$
\tilde{\mathbf{M}}=\frac{1}{K} \mathbf{C C}^{H}
$$


and its corresponding test statistic $\tilde{T}_{N}$

In order to evaluate the robustness of the proposed approach and although the theoretical results are not given in this work, on has also evaluated the detection performance when using the Tyler's estimate [8], [9] for estimating the covariance matrix $\mathbf{M}$, defined as the solution of the following fixed-point equation:

$$
\hat{\mathbf{M}}_{F P}=\frac{N}{K} \sum_{k=1}^{K} \frac{\mathbf{c}_{k} \mathbf{c}_{k}^{H}}{\mathbf{c}_{k}^{H} \hat{\mathbf{M}}_{F P}^{-1} \mathbf{c}_{k}},
$$

where $\mathbf{c}_{k}$ represent the $k^{\text {th }}$ column vector of observation matrix $\mathbf{C}$.

Figures 2(a) and 2(b) display the PFA versus the detection threshold respectively for a SCM-based approach and for a Tyler-based approach. The first remark is that,even for small $N$ and $K$ (here $N=10$ and $K=20$ ), the green curve that represents the proposed approach is very close to the dark one that is the optimal case. This validates the theoretical result given in Corollary 1. Moreover, notice that there is an improvement due to the space whitening effect. Indeed, to guarantee the same level of false alarm, the detection threshold increases in the classical case.

Then, figures 3(a) and 3(b) display the PD versus the SNR of the target, for a PFA of $10^{-2}$. The corresponding detection threshold is obtained thanks to figures 2(a) and 2(b). Again, although no theoretical result is given in this paper (we proved the consistency under $H_{0}$ ), one can see the good agreement between the limiting solution and the empirical one. Then, one can evaluate the gain, that is similar under both SCM and Tyler-based approches: for a PD of 0.9, there is a gain in SNR of almost $3 d B$.

Finally, notice that under both hypotheses $H_{0}$ and $H_{1}$, performance are sometimes better with the proposed approach compared to the T-known case. This can be explained by the fact that $\mathbf{M}$ is also unknown and has to be estimated.

\section{CONCLUSion}

This paper has focused on the joint estimation of joint spatial and temporal covariance matrices arising for adaptive radar detection schemes. This estimation was efficiently performed using latest results coming from RMT with a Toeplitz covariance structure assumption for the spatial covariance matrix and $M$-estimators for the temporal covariance matrix. First results show that the ANMF built with these estimates has significant higher performances, in term of regulation of false alarm and probability of detection versus SNR, than those of the ANMF built with classical estimates supposing erroneously i.i.d. spatially secondary data.

\section{ACKNOWLEDGMENT}

Couillet's work is jointly supported by the French ANR DIONISOS project (ANR-12-MONU-OOO3) and the GDR ISIS-GRETSI-Jeunes Chercheurs-Project. Ovarlez and Pascal's work has been partially supported by the ICODE institute, research project of the Idex Paris-Saclay.

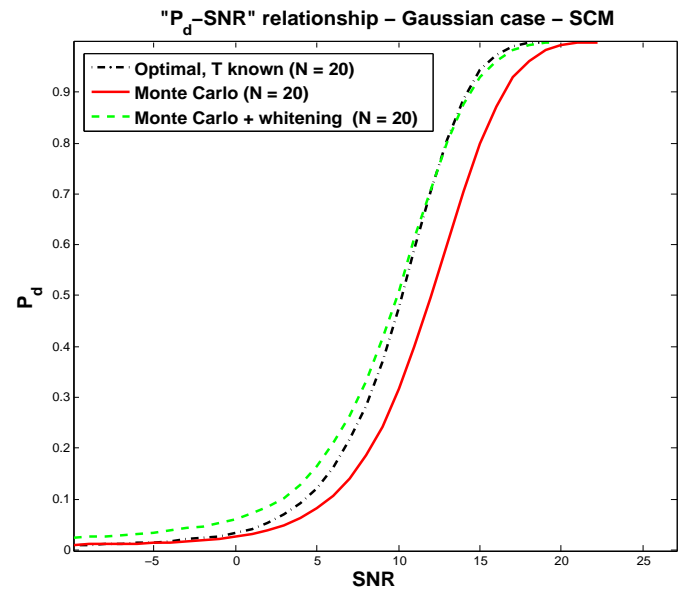

(a) $\mathrm{SCM}$

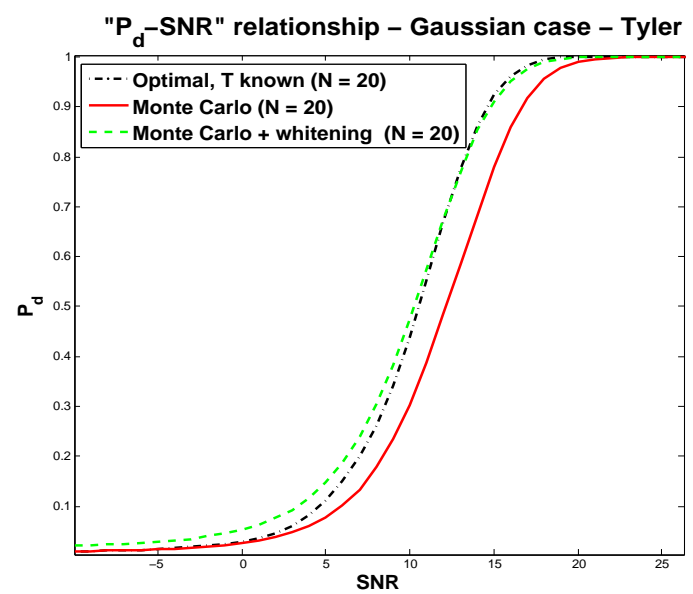

(b) Tyler

Fig. 3. Detection performance under Gaussian noise, $N=10, K=20$.

\section{REFERENCES}

[1] E. Kelly, "An adaptive detection algorithm," Aerospace and Electronic Systems, IEEE Transactions on, vol. AES-22, no. 2, pp. 115-127, March 1986.

[2] S. Haykin and A. O. Steinhardt, Adaptive Radar Detection and Estimation. John Wiley \& Sons, New York, 1992.

[3] M. Greco, F. Gini, and M. Rangaswamy, "Statistical analysis of measured polarimetric clutter data at different range resolutions," Radar, Sonar and Navigation, IEE Proceedings -, vol. 153, no. 6, pp. 473-481, December 2006.

[4] "http://soma.ece.mcmaster.ca/ipix/."

[5] J. Vinogradova, R. Couillet, and W. Hachem, "Estimation of toeplitz covariance matrices in large dimensional regime with application to source detection," arXiv preprint arXiv:1403.1243, 2014.

[6] E. Conte, M. Lops, and G. Ricci, "Asymptotically optimum radar detection in compound-gaussian clutter," Aerospace and Electronic Systems, IEEE Transactions on, vol. 31, no. 2, pp. 617-625, April 1995.

[7] R. Couillet, A. Kammoun, and F. Pascal, "Second order statistics of robust estimators of scatter. Application to GLRT detection for elliptical signals." Journal of Multivariate Analysis (Submitted to), 2015. arXiv: $1410.0817 \mathrm{v} 1$.

[8] D. Tyler, "A distribution-free $m$-estimator of multivariate scatter," The Annals of Statistics, vol. 15, no. 1, pp. 234-251, 1987.

[9] F. Pascal, Y. Chitour, J.-P. Ovarlez, P. Forster, and P. Larzabal, "Covariance structure maximum-likelihood estimates in compound Gaussian noise: existence and algorithm analysis," Signal Processing, IEEE Transactions on, vol. 56, no. 1, pp. 34-48, Jan. 2008. 